\title{
Avalanche-to-Streamer Transition in Particle Simulations
}

\author{
Chao Li, Ute Ebert, and W. J. M. Brok
}

\begin{abstract}
The avalanche-to-streamer transition is studied and illustrated in a particle model. The results are similar to those of fluid models. However, when superparticles are introduced, numerical artifacts become visible. This underscores the need of models that are hybrid in space.
\end{abstract}

Index Terms-Particle simulation, streamers.

$\mathbf{S}$ TREAMERS are growing filaments of weakly ionized nonstationary plasma produced by a sharp ionization front that propagates into nonionized matter. Streamers are used in industrial applications such as lighting [1], ozone generation, and gas and water purification, and they occur in natural processes such as lightning and transient luminous events in the upper atmosphere [2]. Streamers can emerge from ionization avalanches in free space when the self-induced field becomes comparable to the applied field; this was recently reinvestigated in the framework of a fluid model in [3]. Reference [4] discussed that fluid models cannot be applied to avalanches in noble gas in a very low field; and in [5], striations were found in this case. In nitrogen and in intermediate fields, particle and fluid models give essentially the same results for planar ionization fronts, whereas there are growing deviations again at larger fields as discussed in [6].

Here, we show how a streamer emerges in a particle model from few seed electrons and a consecutive avalanche. The system is pure nitrogen at standard temperature and pressure in a constant high field of $100 \mathrm{kV} / \mathrm{cm}$ (equivalent to $42 \mathrm{Td}$ ). We assume that the electrodes are far away or do not exist as in many natural discharges. Qualitatively similar results in a fluid model are shown in [3, Fig. 1].

The particle model includes the complete electron velocity and energy distribution, as well as the discrete nature of particles. However, the required computation resources grow with the number of particles and eventually exceed the limits of any computer. This difficulty can be counteracted by using superparticles carrying the charge and the mass of many physical particles, but superparticles in turn create unphysical

Manuscript received December 1, 2007; revised February 14, 2008. This work was supported by the Dutch national program BSIK in the ICT project BRICKS (theme MSV1).

C. Li is with the Center for Mathematics and Computer Science (CWI), 1090 GB Amsterdam, The Netherlands.

U. Ebert is with the Center for Mathematics and Computer Science (CWI), 1090 GB Amsterdam, The Netherlands, and also with the Department of Applied Physics, Eindhoven University of Technology, 5600 MB Eindhoven, The Netherlands.

W. J. M. Brok is with the Department of Applied Physics, Eindhoven University of Technology, 5600 MB Eindhoven, The Netherlands.

Digital Object Identifier 10.1109/TPS.2008.922487 fluctuations and stochastic heating, as we will demonstrate hereafter.

The simulation starts from 100 pairs of electrons and ions at one point and follows the initial particles and their offspring up to 0.36-ns time. In Fig. 1, the simulated streamer is shown at two different times: $t=0.18 \mathrm{~ns}$ with real particles (first row), $t=0.36 \mathrm{~ns}$ with real particles (second row), and once again $t=0.36 \mathrm{~ns}$, now in a superparticle simulation (third row).

At time $t=0.18 \mathrm{~ns}$ (first row), we have approximately $10^{5}$ electrons, and the maximal field enhancement is $\sim 1 \%$. Therefore, the discharge is still in the avalanche phase. For the second row, de computation is continued with real particles, whereas for the third row, superparticles are introduced as follows. If a specified number of particles, in our case $10^{5}$, is reached, a particle remapping is applied to reduce the number of computational particles; in this step, half of them are thrown away at random, and the weight of the remaining computational particles is doubled. At $t=0.36 \mathrm{~ns}$, the total number of electrons is roughly $1.5 \times 10^{7}$ within the real particle simulation. The space-charge layer has clearly formed. The maximal field enhancement is $\sim 50 \%$. At $t=0.36 \mathrm{~ns}$ within the superparticle simulation, the total number of superparticles is about $7 \times 10^{4}$, each representing 256 real electrons. No charge layer has formed, but the charge density is noisy with a maximum inside the discharge. Clearly, superparticles here are not appropriate to deal computationally with large electron numbers. In [7], we describe how to circumvent the unwieldy runtimes of real particle models by using a hybrid model which couples particle and fluid models in different spatial regions.

\section{REFERENCES}

[1] A. Bhoj and M. Kushner, "Plasma dynamics during breakdown in an HID lamp," IEEE Trans. Plasma Sci., vol. 33, no. 2, pp. 518-519, Apr. 2005.

[2] V. P. Pasko, "Red sprite discharges in the atmosphere at high altitude: The molecular physics and the similarity with laboratory discharges," Plasma Sources Sci. Technol., vol. 16, no. 1, pp. S13-S29, Feb. 2007.

[3] C. Montijn and U. Ebert, "Diffusion correction to the Raether-Meek criterion for the avalanche-to-streamer transition," J. Phys. D, Appl. Phys., vol. 39, no. 14, pp. 2979-2992, Jul. 2006.

[4] B. J. P. Dowds, R. K. Barrett, and D. A. Diver, "Streamer initiation in atmospheric pressure gas discharges by direct particle simulation," Phys. Rev. E, Stat. Phys. Plasmas Fluids Relat. Interdiscip. Top., vol. 68, no. 2, p. 026412 , Aug. 2003.

[5] W. J. M. Brok, "Modelling of transient phenomena in gas discharges," Ph.D. dissertation, Eindhoven Univ. Tech., Eindhoven, The Netherlands, 2005. [Online]. Available: http://alexandria.tue.nl/extra2/200512799.pdf

[6] C. Li, W. J. M. Brok, U. Ebert, and J. J. A. M. van der Mullen, "Deviations from the local field approximation in negative streamer heads," J. Appl. Phys., vol. 101, no. 12, p. 123 305, Jun. 2007.

[7] C. Li, U. Ebert, W. J. M. Brok, and W. Hundsdorfer, "Spatial coupling of particle and fluid models for streamers: where nonlocality matters," J. Phys. D, Appl. Phys., vol. 41, no. 3, p. 032 005, Feb. 2008. 

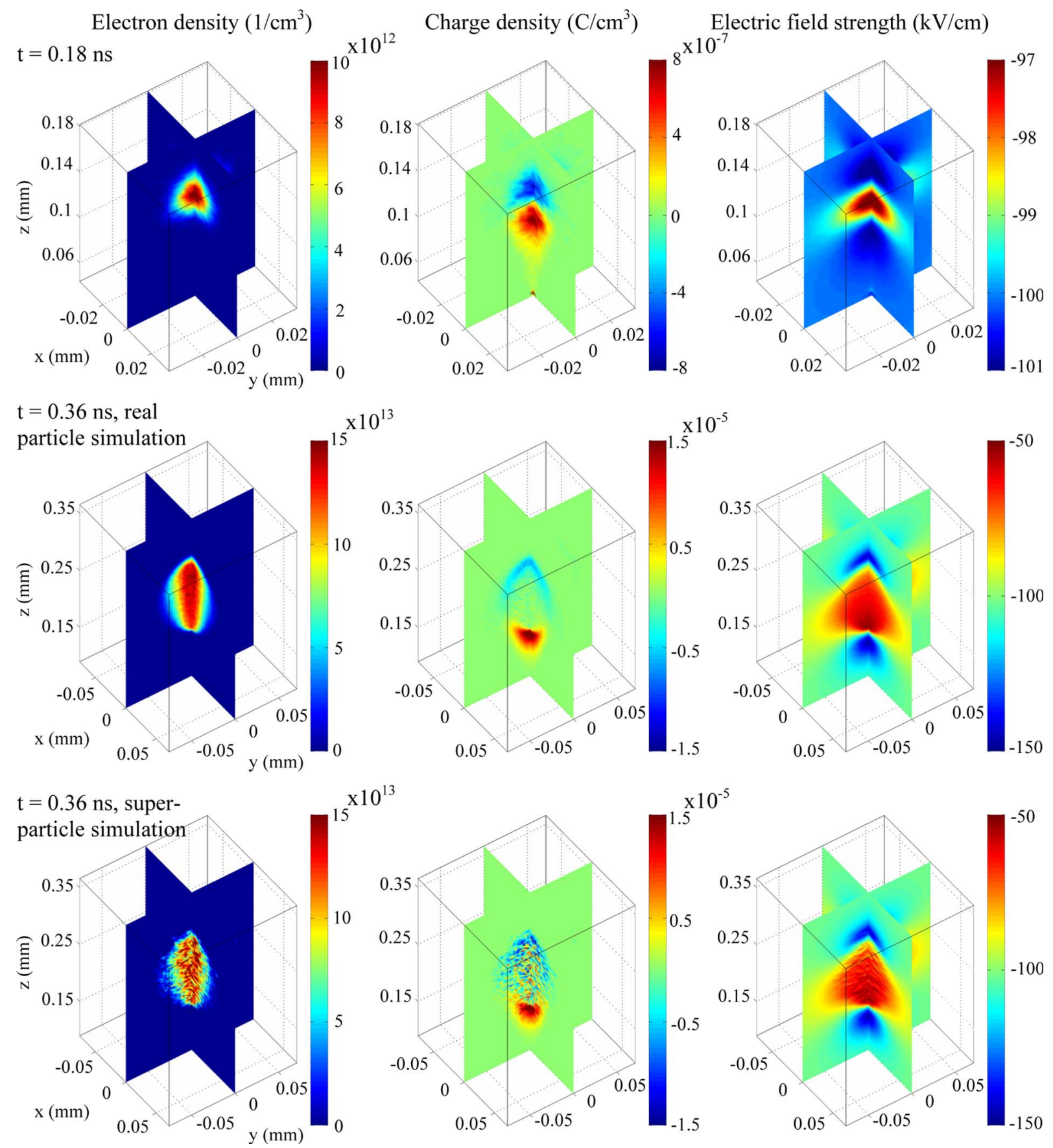

Fig. 1. Avalanche to streamer transition in nitrogen at standard temperature and pressure. A constant background electric field of $100 \mathrm{kV} / \mathrm{cm}$ points downwards. First row: avalanche of real particles at $t=0.18 \mathrm{~ns}$, second row: streamer formed by real particles at $t=0.36 \mathrm{~ns}$, third row: the same system at the same time

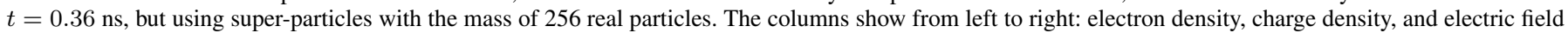
strength. Particle densities and fields are represented on two orthogonal planes that intersect with the 3-D structure. 